\title{
Clima institucional y calidad de gestión educativa en las Instituciones Educativas del Nivel Primario de la provincia de Padre Abad, Ucayali -2019 \\ Institutional climate and quality of educational management in the Primary Level Educational Institutions in the province of Padre Abad, Ucayali -2019
}

\author{
Kely Janeth Rivera Samaniego ${ }^{1}$, Manuel Mamani Flores ${ }^{2}$ y Dina Pari Quispe ${ }^{2}$
}

${ }^{1}$ Unidad de Gestión Local de Padre Abad. Av. Simon Bolivar № 344, Aguaytia, Ucayali, Perú. Email: kelyjanethsamaniego@gmail.com. ORCID: http://orcid.org/0000-0002-7550-0347

${ }^{2}$ Universidad Nacional de Ucayali - Perú. Carretera Federico Basadre Km 5.5, Calleria, Ucayali, Perú. Email. manuel_mamani@unu.edu.pe. ORCID: http://orcid.org/0000-0003-23185581

2 Universidad Nacional de Ucayali. Carretera Federico Basadre Km 5.5, Calleria, Ucayali, Perú. Email: dina_pari@unu.edu.pe. ORCID: http://orcid.org/0000-0002-1493-9209

\section{Resumen}

Se determinó la relación del clima institucional y la calidad de gestión educativa, para ello se trabajó con 171 encuestas cuyo instrumento fue un cuestionario y se realizó de manera transversal, asimismo se realizó la Baremación de los datos. Las dimensiones utilizadas para determinar el clima institucional fueron el liderazgo, la toma de decisiones, el compromiso laboral, la satisfacción laboral y el trabajo en equipo. Para medir la calidad de gestión educativa se utilizó la pedagogía curricular, la administrativa, la organizativa y la comunicativa, obteniendo así la relación que existe entre el liderazgo, la toma de decisiones, el compromiso laboral, la satisfacción laboral y el trabajo en equipo, donde el valor de $r$ fue de 0.39 (p-valor $=0.000 ; p<0.05), 0.393$ (p-valor $=0.000 ; p>0.05), 0.588(p$ valor $=0.000 ; \mathrm{p}<0.05), 0.567(\mathrm{p}$-valor $=0.000 ; \mathrm{p}<0.05) \quad$ y $0.351(\mathrm{p}$-valor $=0.000 ; \mathrm{p}<0.05)$ respectivamente a un nivel de confianza del 95\%. Estos resultados nos muestran que, si existe relación entre el liderazgo, toma de decisiones, compromiso laboral, satisfacción laboral y el trabajo en equipo con la calidad de gestión educativa.

Palabras clave: Gestión; liderazgo; motivación; satisfacción.

\begin{abstract}
The relationship between the institutional climate and the quality of educational management was determined. To this end, 171 surveys were carried out using a questionnaire and a cross-sectional approach, and the data was compiled. The dimensions used to determine the institutional climate were leadership, decision-making, work commitment, work satisfaction and teamwork. To measure the quality of educational management, curricular, administrative, organizational and communicative pedagogy were used, thus obtaining the relationship between leadership, decision-making, work commitment, work satisfaction and teamwork, where the value of $r$ was 0.39 (p-value $=0.000$; $\mathrm{p}<0.05), 0.393(\mathrm{p}$-value $=0.000 ; \mathrm{p}>0.05), 0.588(\mathrm{p}$-value $=0.000 ; \mathrm{p}<0.05), 0.567(\mathrm{p}$-value $=0.000 ;$ $\mathrm{p}<0.05)$ and $0.351(\mathrm{p}$-value $=0.000 ; \mathrm{p}<0.05)$ respectively at a $95 \%$ confidence level. These results show us that there is a relationship between leadership, decision making, work commitment, work satisfaction, and teamwork with the quality of educational management.
\end{abstract}

Keywords: Management; leadership; motivation; satisfaction. 


\section{Introducción}

La gestión educativa es entendida como un proceso organizado y orientado a la optimización de procesos y proyectos internos de las instituciones, con el objetivo de perfeccionar los procedimientos pedagógicos, directivos, comunitarios y administrativos que en ella se movilizan. (Rico, 2016). A nivel educativo la calidad es hoy en día, la bandera de muchos procesos, lo que requiere tratar la manera como se gestiona la calidad en las Instituciones (Cabrera, Beleño, Molina \& Aponte, 2015). Sin embargo la Ley General de Educación, en su artículo 13 establece que la calidad Educativa es el "nivel óptimo de información que debieran alcanzar las personas para hacer frente a los retos del desarrollo humano, ejercer su ciudadanía y aprender a lo largo de toda la vida" (Díaz, 2017).

Por otro lado El clima institucional es un elemento fundamental que debe ser analizado, estructurado y ejecutado por las personas que ejercen la toma de decisiones en las instituciones educativas(Álvarez , 2017), porque no todos los docentes se sienten motivados, para la enseñanza o no todos los estudiante se encuentran motivados para el aprendizaje, es decir es una tarea muy difícil, pero los docentes hacen lo posible para poder cumplir sus metas (Delgado, 2017).

\section{Clima institucional}

El clima institucional es el ambiente generado en una institución educativa a partir de las vivencias cotidianas de los miembros de la Institución Educativa que incluye aspectos como la, relaciones interpersonales, la gestión institucional, estilo de liderazgo, la participación, comunicación y normas (Sardá $\&$ Salgueiro, 2010). Por otro lado se debe considerar las variables del ambiente físico, estructurales, sistema social, personales y propias de la dinámica organizacional (Delgado, 2017). Es decir, se debe de considerar el liderazgo, la toma de decisiones, el compromiso laboral, la satisfacción laboral y el trabajo en equipo.

a. Liderazgo: Emerge en una situación de demanda, necesidad o crisis y se pronuncia con base en el logro de metas, convirtiendo en líder a quien tiene la posibilidad de ofrecer herramientas de empoderamiento (Navarro-Cornoa, 2016); dependiendo no sólo de la capacidad de elección y de influencia ejercitada por una o varias personas, sino también de los horizontes de referencia escogidos y de la capacidad de orientar hacia esos horizontes y conseguir que sean compartidos (Balduzzi, 2015). Por otro lado el liderazgo pedagógico enfatiza en un tipo de liderazgo escolar que tiene propósitos educativos tales como establecer objetivos educativos, planificar el currículum, evaluar a los docentes y la enseñanza y promover el desarrollo profesional docente (Gajardo \& Ulloa, 2016). Por lo tanto el liderazgo educativo es importante a partir de algunos elementos clave que repercuten en el comportamiento del líder (Sierra, 2016). En este sentido el director, tiene que ejercer un papel "transformador": estimular y desarrollar un clima de colegialidad, contribuir al desarrollo profesional de sus profesores, e incrementar la capacidad de la escuela para resolver sus problemas (Antonio, 2010). Asimismo para realizar un trabajo colaborativo es necesario contar con un líder elegido por el mismo equipo que guiará, asegurará que todos los miembros trabajen de manera armoniosa (Lozano Rodriguez \& Tijerina (2013). Porque el mayor liderazgo en los estudiantes acomodadores son los que resaltan en su característica de su estilo como el compromiso, la organización, la aceptación de retos y destacando más que otros estilos para el aprendizaje colaborativo (Arenas et al., 2017). 
b. Toma de decisiones: Es el acto de elegir entre alternativas posibles sobre las cuales existe incertidumbre (Easton, n.d.); asimismo es el proceso mediante el cual se realiza una elección entre las alternativas o formas para resolver diferentes situaciones de la vida, estas se pueden presentarse en diferentes contextos: a nivel laboral, familiar, sentimental, empresarial, etc. (Gutiérrez, 2015). Sin embargo, las buenas decisiones no se logran fácilmente, son el resultado de un arduo y ordenado proceso mental. (Borea \& Vélez, 2018). La capacidad de decidir es la habilidad más genuinamente humana de entre todas ellas. (Mengual, Sempere, Juárez, \& Rodrguez, 2012)

c. Compromiso laboral: Es un fenómeno que se desarrolla y experimenta en un contexto específico, y que se da a través de la interacción entre las personas y su (Santofimio \& Segura, 2005); es decir es el intercambio de acuerdos entre los individuos y la organización (Frías, 2014). El modelo de compromiso laboral enfatiza la perspectiva del largo plazo, con grandes oportunidades de promoción y altos niveles de seguridad para los empleados (Peralta, Santofinio, \& Segura, 2007); permitiendo conseguir buenos resultados y tener los valores y la cultura alineada dentro de una empresa (Mhech , Cordero, \& Gómez, 2016). De esta manera los factores que tienen mayor correlación con el compromiso son: comunicación interna, interés de la dirección, entrenamiento y conciliación entre el trabajo y la familia (Ruiz, 2013).

d. Satisfacción laboral: Es un agradable estado emocional que resulta de la evaluación de un trabajo o la experiencia de un trabajo (Frías, 2014); asimismo, es el grado de conformidad de la persona respecto a su entorno de trabajo (Gonzales, 2018), es un constructo formado por un conjunto de actitudes frente a distintos aspectos relacionados con la situación laboral (Perez, 2011). Por otro lado Hoppck en 1935 indico que los factores que determinan la satisfacción laboral son el equilibrio emocional, religiosidad, sociabilidad, sentimiento de éxito, familia y reconocimiento social, visión del propio trabajo, edad; los factores de personalidad y actitud hacia el entorno (Güell Malet, 2015). Sin embargo Bedoya, Carrilo , Severiche, \& Espinosa ( 2018) precisan que los factores que influyen en la satisfacción laboral son la supervisión y la participación; con el cargo y los factores de ambiente físico y prestaciones y la edad con la participación.

e. Trabajo en equipo: Es el conjunto de personas asignadas o autoasignadas, de acuerdo a habilidades y competencias específicas, para cumplir una determinada meta bajo la conducción de un coordinador (Rivas, 2010). Siendo es una modalidad de articular las actividades laborales de un grupo humano en torno a un conjunto de fines, de metas y de resultados a alcanzar (Instituto Internacional de Planeamineto de la educación, 2000). Sin embargo la competencia de trabajo en equipo se impone a la individualización laboral (Torrelles et al., 2011).

\section{Calidad de gestión educativa}

Gestión educativa es entendida como un proceso organizado y orientado a la optimización de procesos y proyectos internos de las instituciones, con el objetivo de perfeccionar los procedimientos pedagógicos, directivos, comunitarios y administrativos que en ella se movilizan. La institución es autónoma de definir sus criterios de gestión más adecuados y encaminados a responder con las necesidades educativas resultantes de la comunidad, más las exigencias legales de corte nacional e internacional. La gestión

Downloadable from: http://revistas.unu.edu.pe Carretera Federico Basadre $\mathrm{Km} 6$, Dirección de Producción Intelectual

Revista de Investigación Universitaria por Universidad Nacional de Ucayali se distribuye bajo una Licencia Creative Commons Atribución-NoComercial 4.0 Internacional. 
educativa se convierte en una disciplina necesaria para ejercer la dirección y el liderazgo integral en las organizaciones educativas y para lograr el cumplimiento de su función esencial: la formación integral de la persona y del ciudadano, de manera que logre insertarse creativa y productivamente en el mundo laboral (Rico, 2016).

Asimismo es el conjunto de acciones orientadas hacia la consecución de ciertos objetivos que se desarrollan en las diversas áreas de actividad de la organización y en cuyo diseño y evaluación participan, en alguna medida, las personas encargadas de llevarlas a cabo (García et al., 2018).

A nivel educativo la calidad es hoy en día, la bandera de muchos procesos, lo que requiere tratar la manera como se gestiona la calidad en las Instituciones, es decir se hace evidente entonces, la necesidad de tratar teóricamente, qué es Gestión. Esta y calidad van unidas, ya que a través de la primera se puede determinar la segunda, la gestión se ejerce, la calidad es el resultado, van de la mano; se gestiona para alcanzar un mejoramiento continuo de todos aquellos aspectos que ponen en funcionamiento la institución y a través de los cuales se llega a lograr lo visionado (Cabrera, Beleño, Molina \& Aponte, 2015).

\section{Metodología}

El trabajo de investigación es básico o pura, transversal, descriptiva, cuantitativa de campo cuya orientación es de comprobación (Córdova, 2014). La investigación es transversal correlacional (Gómez, 2013; Hernandez, Zapata, \& Mendoza, 2013). Asimismo la valoración del grado de correlación utilizada fue el establecido por Hernandez-Sapieri \& Mendoza Torres (2018) quienes establecieron que los coeficientes de correlación pueden variar de cero a uno ( 0 a 1$)$ pudiendo obtenerse correlaciones negativas o positivas, estos pueden ser: muy fuerte, considerable, media, débil, muy débil y no puede existir correlación alguna entre las variables. El esquema del diseño es el siguiente:

La población, objeto de estudio, está constituido por todos los directivos (8), docentes (175) y administrativos (6) de las Instituciones Educativas del Nivel Primario $\mathrm{N}^{\circ}$ 64027, 64043, 64114, 64109, 64109, 64052 y 64723 de la provincia de Padre Abad, 2019.

La técnica que se utilizó para la recolección de datos fue la encuesta, cuyo instrumento fue el cuestionario. Para lo cual se construyó dos cuestionarios, uno para la variable clima institucional (V1) y otro para medir la variable Calidad de gestión educativa (V2). Ambos cuestionarios fueron de tipo Likert con preguntas cerradas cuyas alternativas de respuesta para cada ítem fue: Nunca, casi nunca, alguna vez, casi siempre y Siempre. Estas alternativas de respuesta para el tratamiento estadístico tuvieron una escala numérica de 1, 2, 3, 4, 5 la que nos facilitó el análisis respectivo.

Después de la recolección de la información, independientemente de las fuentes utilizadas, se organizó los datos para presentar los resultados y su posterior análisis. Las respuestas de cada encuesta fueron ingresadas en la base de datos de Excel 2013 debidamente organizados. Los resultados fueron obtenidos mediante la aplicación de la estadística descriptiva o inferencial. Para conocer el grado de relación de las variables se utilizó la $r$ de Pearson.

\section{Resultados y discusiones}

El liderazgo emerge en una situación de demanda, necesidad o crisis y se pronuncia con base en el logro de metas, convirtiendo en líder a quien tiene la posibilidad de ofrecer herramientas de empoderamiento (NavarroCornoa, 2016). 


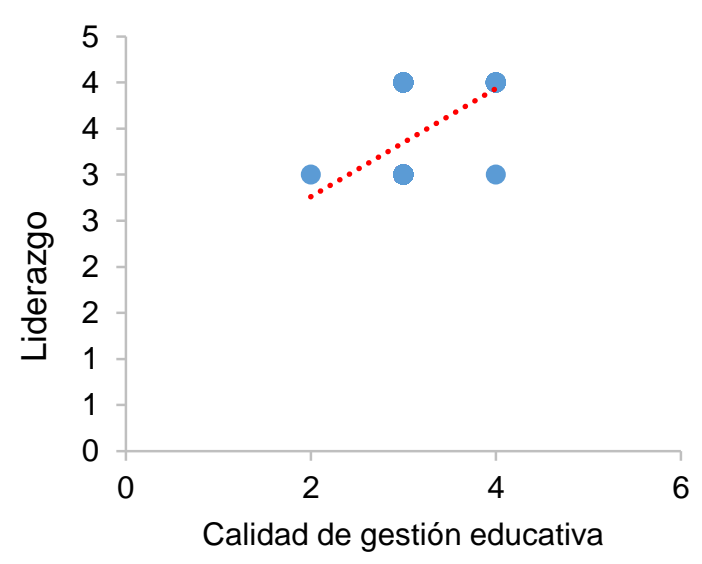

Figura 1: Relación del liderazgo y la calidad de gestión educativa de las Instituciones Educativas de Nivel Primario de la Provincia de Padre Abad

El liderazgo de los directivos de las diferentes instituciones educativas del nivel primario de la provincia de Padre Abad guarda una relación moderada con la calidad de gestión educativa puesto que permite mejorar la pedagogía curricular, la administrativa, la organizativa y la comunicativa, asimismo los resultados nos muestran que el valor de " $r$ " de Pearson es de 0.39 , este valor evidencia que existe una relación positiva débil de las variables, asimismo el p-valor $=0.000$; $\mathrm{p}<0.05$, siendo significativo, tal como se muestra en la figura 01. Estos resultados coinciden con lo manifestado por Atalaya (2016) y Espejo (2017) quienes precisan que el liderazgo del director es fundamental para mejorar la calidad de la educación así como también para mejorar el desempeño docente. Asimismo Briones-Álvarez (2017) encontró que el liderazgo es positivo. Por otro lado el liderazgo pedagógico enfatiza en un tipo de liderazgo escolar que tiene propósitos educativos tales como establecer objetivos educativos, planificar el currículum, evaluar a los docentes y la enseñanza y promover el desarrollo profesional docente (Gajardo \& Ulloa, 2016). En este sentido el director, tiene que ejercer un papel transformador como estimular y desarrollar un clima de colegialidad (Antonio, 2010).

Uno de los factores muy importantes para una calidad de gestión educativa es la toma de decisiones, puesto que permite elegir de manera correcta la de una decisión.

Además los resultados muestran que el valor de "r" de Pearson es de 0,393 (p-valor = 0.000; $\mathrm{p}<0.05$ ), siendo significativo, puesto que si existe una relación positiva débil entre la toma de decisiones de los directivos con la calidad de gestión educativa en las instituciones educativas del nivel primario de la provincia de Padre Abad, pero esta relación es débil, porque tal vez algunas decisiones que toma el director no son apoyadas por los docentes, razón por la cual la toma de decisiones es buena en un alto porcentaje, por lo tanto se acepta la hipótesis alterna y se rechaza la hipótesis nula (figura 02). Es decir la toma de decisiones en una organización invade cuatro funciones administrativas que son: planeación, organización, dirección y control (Gutiérrez, 2015).

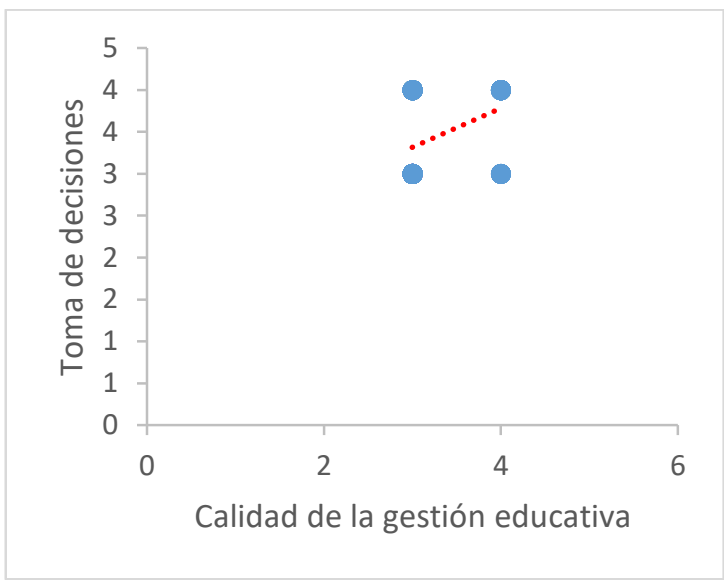

Figura 2: Relación de la toma de decisiones y la Calidad de gestión de las Instituciones Educativas de Nivel Primario de la Provincia de Padre Abad 
Nuestros resultados coinciden con los de Paredes (2015) quien reporto que la toma de decisiones influye significativamente en la calidad de la gestión (Sandoval Bustos \& Diaz Vicuña, 2014). Asimismo se debe de tomar en cuenta los efectos futuros, la reversibilidad, el impacto (Richard, n.d.), esto quiere decir que muchos de los directivos de las instituciones educativas por lo general toman decisiones a corto plazo, razón por la cual no pueden obtenerse resultados significativos (Borea \& Velez, 2018).

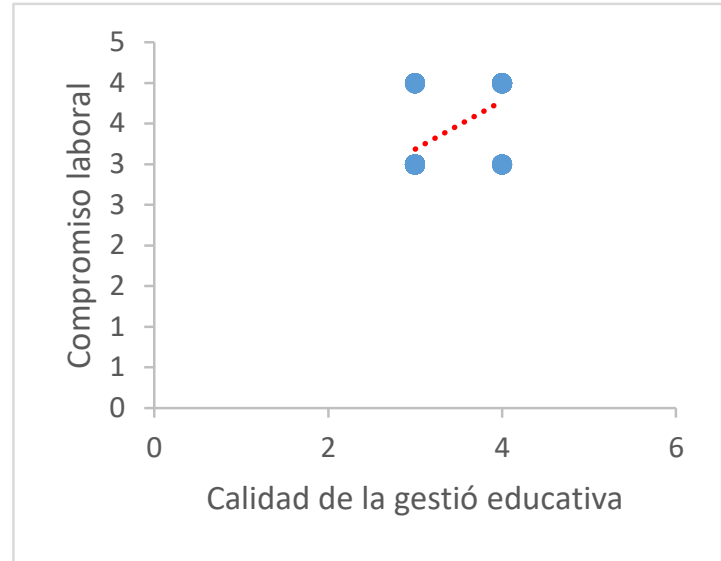

Figura 3: Relación del compromiso laboral y la Calidad de gestión de las Instituciones Educativas de Nivel Primario de la Provincia de Padre Abad

Por otro lado el compromiso laboral de los directivos, docentes y administrativos es muy importante para realizar una calidad de gestión educativa por lo tanto los resultados nos muestran que el valor de " $r$ " de Pearson es de $0,588$ ( $\mathrm{p}$-valor $=0.000 ; \mathrm{p}<0.05)$, siendo significativo, puesto que si existe una relación significativa entre el compromiso laboral de los directivos, docentes y administrativos con la calidad de gestión educativa en las instituciones educativas del nivel primario de la provincia de Padre Abad, pero esta relación es positiva media, por lo tanto se acepta la hipótesis alterna y se rechaza la hipótesis nula (figura 03). Nuestros resultados coinciden con Briones-Álvarez (2017) donde encontró que las habilidades sociales, los niveles de comunicación y la planificación influyen significativamente en la calidad del servicio que brinda la institución educativa. Pero Calcina (2013) reporto que la relación que existe entre el clima institucional y el desempeño laboral de los docentes de la Facultad de Ciencias Sociales de la UNA PUNO, 2012; UANCV - JULIACA, 2012, es positiva alta, con una puntuación de $0.77 \mathrm{y}$ 0.650 respectivamente.

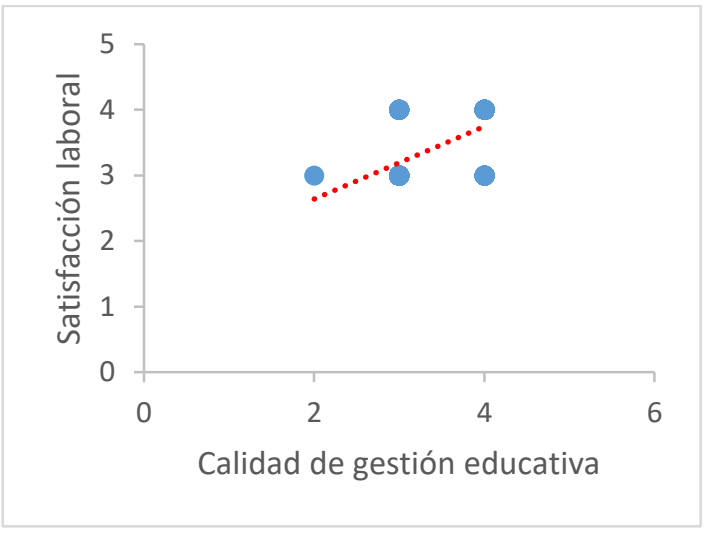

Figura 4: Relación de la satisfacción laboral y la Calidad de gestión de las Instituciones Educativas de Nivel Primario de la Provincia de Padre Abad

En la figura 04 se puede observar la relación de la satisfacción laboral y la calidad de la gestión educativa en las Instituciones de Educativas de Nivel Primario de la provincia de Padre Abad siendo una correlación positiva media, es decir que a calidad de la gestión educativa dependerá mucho del nivel de satisfacción de los docentes y administrativos de las instituciones educativas ya que el valor de " $r$ " de Pearson es de 0.567 , este valor evidencia que existe una relación positiva media de las variables, asimismo el $\mathrm{p}$-valor $=$ $0.000 ; \quad \mathrm{p}<0.05$, siendo significativo, existiendo una relación positiva significativa, 
por lo tanto se acepta la hipótesis alterna y se rechaza la hipótesis nula.

Nuestros resultados concuerdan con lo mencionado por Calvo (2014) quien indica que gracias al diseño e implementación de la estrategia basado en talleres, compuestos por discusiones, cuestionarios y actividades lúdicas, ha sido posible mejorar y profundizar los conocimientos que los docentes tienen sobre los factores que inciden en un adecuado clima institucional; puesto que la satisfacción laboral, es un constructo formado por un conjunto de actitudes frente a distintos aspectos relacionados con la situación laboral (Perez, 2011). Por otro lado, Hoppck en 1935 indico que los factores que determinan la satisfacción laboral son el equilibrio emocional, religiosidad, sociabilidad, sentimiento de éxito, familia y reconocimiento social, visión del propio trabajo, edad. Asimismo la personalidad y actitud hacia el entorno es sumamente importante (Güell Malet, 2015). Sin embargo Bedoya, Carrilo, Severiche, \& Espinosa ( 2018) precisan que los factores que influyen en la satisfacción laboral son la asociación con el nivel académico y los factores supervisión y la participación; con el cargo y los factores de Ambiente físico y prestaciones y la edad con la participación. Por otro lado, el nivel de satisfacción laboral de docentes obtenido se puede catalogar de medio-alto. Además, (Muñoz-Méndez et al., 2017). Pero Cantón \& Téllez (2016) encontraron una pluralidad de enfoques y perspectivas dependiendo del origen científico de cada investigador.

En la figura 05 se puede observar la relación del trabajo en equipo y la calidad de gestión de la Instituciones Educativas del Nivel Primario dela provincia de Padre Abad siendo una correlación positiva débil, es decir que el trabajo en equipo de los directivos, docentes y administrativos no siempre garantizara que exista una calidad en la gestión educativa de las instituciones educativas ya que el valor de "r" de Pearson es de 0..351, este valor evidencia que existe una relación positiva débil o moderada de las variables, asimismo el $\mathrm{p}$-valor $=0.000 ; \mathrm{p}<0.05$, siendo significativo, existiendo una relación positiva significativa, por lo tanto se acepta la hipótesis alterna y se rechaza la hipótesis nula. Nuestros resultados evidencian que puede existir motivación, comunicación, compromiso de los directivos, pero esto no garantiza que tenga una relación positiva alta con la calidad de gestión educativa puesto que el trabajo en equipo va mucho más allá.

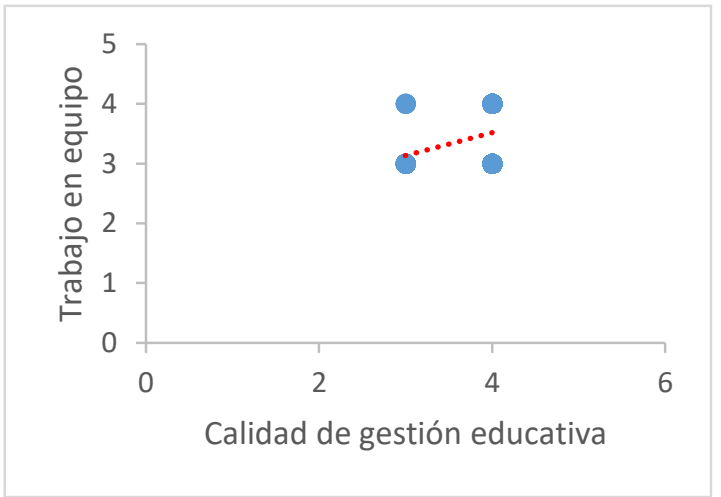

Figura 5: Relación del Trabajo en equipo y la calidad de gestión de las Instituciones Educativas de Nivel Primario de la Provincia de Padre Abad

Por otro lado el trabajo en equipo es una modalidad de articular las actividades laborales de un grupo humano en torno a un conjunto de fines, de metas y de resultados a alcanzar (Instituto Internacional de Planeamineto de la educación, 2000). Tal vez los resultados obtenidos en nuestro trabajo evidenciaron que a veces el trabajo en equipo tiene un interés individual razón por la cual no se puede lograr la calidad de la gestión educativa. Sin embargo la competencia de trabajo en equipo se impone a la individualización laboral (Torrelles et al., 2011).

Downloadable from: http://revistas.unu.edu.pe Carretera Federico Basadre Km 6, Dirección de Producción Intelectual

Revista de Investigación Universitaria por Universidad Nacional de Ucayali se distribuye bajo una Licencia Creative Commons Atribución-NoComercial 4.0 Internacional. 


\section{Conclusiones}

Existe relación positiva entre el clima institucional y la calidad de gestión educativa pero moderada, se tienen que seguir trabajando para obtener una calidad en la gestión educativa en las instituciones educativas de la provincia de Padre Abad, puesto que esto dependerá mucho del liderazgo la toma decisiones, el compromiso y la satisfacción laboral, para ello los directivos de la diferentes instituciones educativas, deben de buscar estrategas para mejorar el clima institucional y así mejorar la calidad de la gestión educativa en la provincia de Padre Abab.

\section{Referencias bibliográficas}

Álvarez Valencia, F. A. (2017). El clima institucional como factor determinante en el rendimiento académico de los alumnos de la Institución Educativa Técnico Industrial Simona Duque del Municipio de Marinilla. Universidad de Antioquia.

Antonio, B. (2010). El liderazgo educativo y su papel en la mejora: una revisión actual de sus posibilidades y limitaciones. revista psicoperpectivas, 9(2), 933 . https://doi.org/10.2225/psicoperspectiva s-vol9-issue2-fulltext-1

Arenas Madroñero, C. E., Tamez Almaguer, R., \& Lozano Rodríguez, A. (2017). Los estilos de aprendizaje y su relación con el aprendizaje colaborativo en cursos virtuales. Revista de Estilos de Aprendizaje, 10(20), 300-320. http://scholar.google.com/scholar?start= 40\&hl=en\&num=20\&as_sdt=2005\&sci odt $=0,5 \&$ cites $=15778326373459255014$ $\&$ scipsc $=\# 8$

Atalaya Jamanca, O. F. (2016). Liderazgo del director y el clima institucional del Consorcio Educativo UGEL 07 - 2013.
Universidad Nacional Mayor de San Marcos.

Balduzzi, E. (2015). Liderazgo educativo del profesor en el aula y la personalización educativa. Revista Española de Pedagogía, 260, 141-155.

Bedoya Marrugo, E., Carrilo Landazabal, M., Severiche Sierra, C., \& Espinosa Fuentes, E. (2018). Factores asociados a la satisfacción laboral en docentes de una institución de educación superior del Caribe Colombiano. Revista Espacios, 39(2), 1-14.

Borea, F., \& Velez pareja, I. (2018). Modulo introductoria teoría de la decisión (p. 34).

Briones-Álvarez, M. (2017). Influencia del clima institucional en la calidad del servicio educativo de la Institución Educativa Ramón Castilla N ${ }^{\circ} 063$, Cajamarca. Revista Perspectiva, 18(1), 27-33.

Cabrera Tafur, J., Beleño de Castro, N., Molina Padilla, G., \& Aponte Herrera, L. (2015). Calidad Educativa y Gestión Escolar.

Calcina Calcina, Y. (2013). El clima institucional y su incidencia en el desempeño laboral de los docentes de la Facultad de Ciencias Sociales de la Universidad Nacional del Altiplano y Facultad de Ciencias de la Educación Universidad Andina Néstor Cáceres Velásquez-Perú 2012. Revista Comunicación, 1(1), 20-29.

Calvo Estrada, S. A. (2014). Estrategia de gestión educativa para fortalecer el clima organizacional de la comuna de docentes y administrativos del colegio instituto Bogotá de ciudad Berna. Universidad Libre.

Cantón Mayo, I., \& Téllez Martinez, S. (2016).

Downloadable from: http://revistas.unu.edu.pe Carretera Federico Basadre $\mathrm{Km}$ 6, Dirección de Producción Intelectual 
La satisfacción laboral y profesional de los profesores. Revista Lasallista de Investigación, 13(1), 214-226.

Córdova Baldeón, I. (2014). El informe de investigación cuantitativa. San Marcos.

Delgado Ramos, G. (2017). Participación y clima institucional para una organización escolar efectiva (Ministerio de la Educación (ed.); Primera Ed).

Diaz Vasquez, S. L. (2017). Calidad de la gestión educativa en el marco del proceso de la acreditación en las instituciones educativas estatales nivel secundario, zona urbana distrito de Iquitos. Universidad Nacional de la Amazonía Peruana.

Easton, D. (n.d.). TEORíAS DE LA TOMA DE DECISIONES.

Espejo Rivera, R. (2017). Gestión institucional y desempeño docente en la Institución Educativa Libertador José de San Martin, UGEL 14, Oyón. Universidad Nacional de Educación Enrique Gusmán y Valle.

Frías Castro, P. (2014). "Compromiso y satisfacción laboral como factores de permanencia de la generación Y ." Universidad de Chile.

Gajardo, J., \& Ulloa, J. (2016). Liderazgo Pedagógico, Conceptos y tensiones. En Lideres Educativos.

Garcia Colina, F., Juárez Hernandez, S., \& Salgado Garcia, L. (2018). Gestión escolar y calidad educativa. Revista Cubana de Educación Superior, 2, 206216.

Gómez Briceño, E. (2013). Investigación científica: Elaboración del proyecto de investigación (A. Sevillano Cueva (ed.)). A.F.A. Editores Importadores S.A.

Gonzales Sánchez, M. (2018). Nivel de
Satisfacción Laboral en el Rol Docente y su Relación con el Síndrome de Desgaste Profesional en los docentes de Colegios Privados. Estudio de Exploración. Universidad Metropolitana.

Güell Malet, L. (2015). Estudio de la satisfacción laboral de los maestros. Universidad Internacional de Catalunya.

Gutiérrez Díaz, A. (2015). Toma de decisiones (p. 15).

Hernandez-Sapieri, R., \& Mendoza Torres, C. P. (2018). Metodología de la investigación, las rutas cuantitativa, cualitativa y mixta. McGraw Hill.

Hernandez Sampiere, R., Zapata Salazar, N. E., \& Mendoza Torres Cristian Paulina. (2013). Metodología de la investigación para bachilelrato (McGRAWHLL/INTERAMERICANA EDITORES S.A. DE C.V. (ed.)).

Instituto Internacional de Planeamiento de la educación. (2000). Trabajo en equipo. In Diez módulos destinados a los responsables de los procesos de transformación educativa (p. 30).

Lozano Rodriguez, A., \& Tijerina Salas, A. (2013). La colaboración en espacios virtuales a través de estilos de aprendizaje desde la perspectiva docente: un estudio de caso. Journal of Learning Styles, 6(11), 38-50. http://revistaestilosdeaprendizaje.com/ar ticle/view/970/1678

Mengual Recuerda, A., Sempere Ripoll, F., Juárez Varón, D., \& Rodríguez Villalobos, A. (2012). El proceso de la toma de decisiones como habilidad directiva. Revista de Investigación Ciencias, 14.

Mhech Vargas, C., Cordero Unghiatti, A., \& Gómez Rabagliati, T. (2016). Medición 
del compromiso laboral y su impacto en los resultados de la empresa.

Muñoz-Méndez, T., Gómez-Mármol, A., \& Sánchez-Alcaraz, B. (2017). Satisfacción laboral en los docentes de educación infantil, primaria y secundaria. Revista Gestión de la Educación, $\quad 7(1), \quad$ 161-177. https://doi.org/DOI: http://dx.doi.org/10.15517/rge.v7i1.275 78

Navarro-Cornoa, C. (2016). Consideraciones teóricas sobre el concepto de liderazgo y su aplicación en la investigación educativa. Revista Educación, 40(1), 53 66.

Paredes Díaz, T. (2015). Relación del clima institucional y la calidad de la gestión, en la Institución Educativa $N^{\circ} 16577$, del centro poblado Montango, distrito de Santa Rosa, Provincia de Jaén, 2014. Universidad Nacional de Cajamarca.

Peralta Gomez, M. C., Santofinio, A. M., \& Segura, V. (2007). El compromiso laboral: discursos en la organización. Psicología Desde El Caribe, 19, 81-109.

Perez Vilar, S. (2011). Satisfacción laboral. Universidad Abierta Interamericana.

Richard, B. (n.d.). Toma de decisiones y solución de problemas (p. 28).

Rico Molano, A. D. (2016). La gestión educativa: Hacia la optimización de la formación docente en la educación superior en Colombia. Revista SOPHIA, 12(1), 55-70.

Rivas Tovar, L. (2010). Trabajo de equipo (p. 39).

Ruiz De Alba, J. Luis. (2013). El compromiso organizacional: un valor personal $\mathrm{y}$ empresarial en el marketing interno. Revista de Estudios Empresariales, 1(1), 67-86.

Sandoval Bustos, E., \& Diaz Vicuña, E. (2014). Procesos de toma de decisiones y adaptación al cambio climático (p. 20).

Santofimio Vengoechea, A. M., \& Segura Trujillo, V. (2005). Significado del compromiso laboral para una organización y para sus empelados.

Sardá, M., \& Salgueiro, A. (2010). Cuadernos para pensar, hacer y vivir la escuela: Clima institucional en la escuela (Proyecto Reforma y fortalecimiento de la gestión del sistema educativo (ed.)).

Sierra Villamil, G. (2016). Liderazgo educativo en el siglo XXI, desde la perspectiva del emprendimiento sostenible. Rev. Esc. Adm. Neg, 81, 111-128.

Torrelles, C., Coiduras, J., Isus, S., Carrera, X., Paris, G., \& Cela, J. (2011). Competencia de trabajo en equipo. Profesorado. Revista de Curriculum y Formación de Profesorado, 15(3), 329-344. 